\title{
Clinical application of a digital thoracic drainage system for objectifying and quantifying air leak versus the traditional vacuum system: a retrospective observational study
}

\author{
Song Am Lee ${ }^{1 \wedge}$, Jun Seok Kim^${ }^{1 \wedge}$ Hyun Keun Chee ${ }^{1 \wedge}$, Jae Joon Hwang ${ }^{1 \wedge}$, Michael $\mathrm{Ji}^{2} \wedge$, Yo Han $\mathrm{Kim}^{3} \wedge$, \\ Hyeong Ju Moon ${ }^{3 \wedge}$, Woo Surng Lee ${ }^{3 \wedge}$ \\ ${ }^{1}$ Department of Thoracic and Cardiovascular Surgery, School of Medicine, Konkuk University, Konkuk University Seoul Hospital, Seoul, Republic \\ of Korea; ${ }^{2}$ Department of Critical Care Medicine, University of Calgary, Calgary, Alberta, Canada; ${ }^{3}$ Department of Thoracic and Cardiovascular \\ Surgery, School of Medicine, Konkuk University, Konkuk University Chungju Hospital, Chungju-si, Chungbuk, Republic of Korea \\ Contributions: (I) Conception and design: WS Lee, JJ Hwang, YH Kim, CK Chee; (II) Administrative support: SA Lee, JS Kim, WS Lee, JJ Hwang, \\ M Ji, CK Chee; (III) Provision of study materials or patients: WS Lee, JS Kim, HJ Moon; (IV) Collection and assembly of data: WS Lee, JS Kim, \\ HJ Moon; (V) Data analysis and interpretation: SA Lee, WS Lee, M Ji; (VI) Manuscript writing: All authors; (VII) Final approval of manuscript: All \\ authors. \\ Correspondence to: Woo Surng Lee, MD, PhD. Professor, Department of Thoracic and Cardiovascular Surgery, School of Medicine, Konkuk \\ University, Konkuk University Chungju Hospital, 82, Gugwon-daero, Chungju-si, Chungbuk, Republic of Korea. Email: timesgoby@naver.com.
}

\begin{abstract}
Background: Digital thoracic drainage systems have recently been introduced and widely used in clinical practices in developed countries. These systems can monitor intrathoracic pressure changes and air leaks in real time, and also allow for objective and quantitative analyses, which aid in managing patients with a prolonged persistent air leak into the pleural space. We investigated the feasibility and effectiveness of such a new device versus the traditional vacuum system for treating patients with pneumothorax.

Methods: Closed thoracostomy drainage was carried out on 100 adult patients with primary or secondary pneumothorax between January 2017 and December 2018. All the patients were aged $\geq 18$ years and treated with a chest tube at a single medical center by the same cardiothoracic surgeons and intensivists. Patients who underwent closed thoracostomy drainage using an indwelling 24-French chest tube were divided into 2 groups immediately before closed thoracostomy: the digital thoracic drainage group (digital group, $\mathrm{n}=50$ ) and the traditional analogue thoracic drainage group (analogue group, $n=50$ ). The detailed information about demographic data, treatment outcome, duration of indwelling catheterization., hospital days, costeffectiveness and patient satisfaction was evaluated. We also evaluated whether digitally recorded intrapleural pressure changes and air leaks would predict chest tube removal timing and outcome.

Results: The baseline parameters of the 2 groups were comparable with no significant differences in sex, age, weight or body mass index. The mean hospital day was shorter in the digital group than in the analogue group $(17.96 \pm 12.23$ vs. $18.32 \pm 16.64, \mathrm{P}=0.902)$, and there was no statistically significant difference in the hospital length of stay between the 2 groups. Air leaks through the chest tube and duration of chest tube indwelling hours showed no significant statistical differences between the digital and analogue groups (213.47 \pm 219.80 vs. $261.94 \pm 184.47, \mathrm{P}=0.235$ and $223.44 \pm 218.75$ vs $275.29 \pm 186.06, \mathrm{P}=0.205$, respectively). Total drainage amount and ambulation time per day were significantly higher in the digital group than in the analogue group $[209.62 \pm 139.63$ vs. $162.48 \pm 80.42(\mathrm{P}=0.042)$ and $6.42 \pm 3.62$ vs.3.94 $\pm 1.74(\mathrm{P}<0.001)$, respectively]. Hours of full expansion were significantly shorter and sleep disturbance caused by the noise of chest tube drainage was less in the digital group than in the analogue group $[25.64 \pm 14.55 v s .46 .52 \pm 25.53$
\end{abstract}

^ ORCID: Song Am Lee, 0000-0002-2218-5849; Jun Seok Kim, 0000-0002-2547-9674; Hyun Keun Chee, 0000-0001-7041-352x; Jae Joon Hwang, 0000-0002-5566-4037; Michael Ji, 0000-0002-4850-2793; Yo Han Kim, 0000-0002-5928-0727; Hyeong Ju Moon, 0000-00029377-6080; Woo Surng Lee, 0000-0002-5691-3584. 
$(\mathrm{P}<0.001)$ and $2.38 \pm 1.03$ vs. $5.70 \pm 2.87(\mathrm{P}<0.001)$, respectively].

Conclusions: To date, there is no definite consensus and guidelines on the standardized digital suction system in pneumothorax. This study proposed the guidelines for the application of digital thoracic drainage systems in pneumothorax and also suggested that digital thoracic drainage systems might be a valuable tool to determine chest tube removal timing and reducing the length of hospital stay in patients with pneumothorax.

Keywords: Thoracostomy; chest tubes; drainage; suction; pneumothorax; digital; analogue

Submitted Sep 28, 2020. Accepted for publication Dec 18, 2020.

doi: $10.21037 /$ jtd-20-2993

View this article at: http://dx.doi.org/10.21037/jtd-20-2993

\section{Introduction}

In the fifth century BCE, the main concept of chest drainage was first advocated by Hippocrates, and he described pleural drainage with a thoracic incision, cautery and insertion of a metal tube for the treatment of empyema. In the 15 th century CE, significant advances in the treatment of empyema include rib resection and insertion of a trocar and a metal cannula for thoracic drainage, which was described by Celsius, and these instruments were completely similar to those available nowadays (1-3). In 1871, a British physician, William Smoult Playfair, reported on the application of continuous subaqueous drainage to treat thoracic empyema in children, and established the principle of the current underwater-seal suction drainage. He was faced with a patient having an open pneumothorax created by tube thoracostomy, who was successfully treated with continuous subaqueous drainage of the pleural space using a one-compartment bottle system with direct connection to the thoracic drainage tube and a rigid straw immersed approximately $2.0 \mathrm{~cm}$ below water which plays an important role as both a one-way valve and a venting valve (4). His novel idea using a onecompartment bottle system offered a safe and complete egress of air from the pleural cavity to atmosphere during inspiration and prevented a re-entry of air on expiration. In 1875, a German internist and pulmonologist, Gotthard Bülau, demonstrated a more modernized closed water seal drainage system for the treatment of pleural empyema (5). To date, digital chest tube drainage systems (DCTS) have contributed to quantifying air leaks and determining when to remove chest tubes. Previous studies have demonstrated that compared to traditional systems, these systems aid in clinical determination of chest drain removal timing and further reduce the length of hospital stay in patients with air leaks, which makes these systems useful for managing patients with primary or secondary pneumothorax in a clinical setting. In the field of cardiothoracic surgery, most patients with air leaks into the pleural space should be managed by placement and maintenance of chest tubes. However, the placement and management of chest tubes are relatively complex due to considerable variations and debates among different institutions, especially in a clinical setting $(6,7)$. There are some debates on (I) when chest tubes should be removed in patients with radiologically resolved pneumothorax, (II) whether chest tube suction is helpful in shortening the chest tube maintenance, and (III) whether provocation test by chest tube clamping is useful for determining the optimal timing of removal (7-11). This retrospective study was conducted to evaluate the feasibility and usefulness of a digital thoracic drainage system in patients admitted with pneumothorax at our institution. We present the following article in accordance with the STROBE reporting checklist (available at http://dx.doi. org/10.21037/jtd-20-2993).

\section{Methods}

\section{Study subjects}

Between January 1, 2017 and December 31, 2018, we recruited 100 patients who were diagnosed with primary or secondary pneumothorax and underwent closed thoracostomy drainage using a 24-French chest tube at the Department of Thoracic and Cardiovascular Surgery and the Department of Intensive Care Medicine. Patients aged between 18 and 80 years with chest tube drains for the treatment of pneumothorax were eligible for the study. Patients who underwent closed thoracostomy drainage using an indwelling chest tube were divided into 2 groups by an institutional review board moderator who was blinded to the study using block randomization with blocks of 
4 immediately before closed thoracostomy: those receiving thoracic suction drainage using a digital drainage device (digital group, $\mathrm{n}=50$ ) and those receiving thoracic suction drainage using a traditional drainage device (analogue group, $\mathrm{n}=50$ ). All surgical procedures and managements were performed by a single team consisting of the same thoracic surgeon, intensivist and traumatologist as well as nurse and physician assistant. The detailed information about demographic data, treatment outcome, duration of indwelling catheterization, hospital days, cost effectiveness and patient satisfaction was evaluated. We also evaluated whether digitally recorded intrapleural pressure changes and air leaks would predict chest tube removal timing and outcome.

\section{Study design}

The aim of this study was as follows: (I) to compare effectiveness between chest drainage systems using a digital drainage device and a traditional water seal suction device in the management of patients with primary or secondary pneumothorax; (II) to collect data about digitally recorded intrapleural pressure changes and air leaks in order to determine whether the data would predict treatment outcome, when the chest drain should be removed, and whether the patient would require surgery; and (III) to more objectively assess the satisfaction of surgeons, physicians and nurses with the use of this digital drainage device compared to a traditional underwater-seal suction device in patients with pneumothorax .

\section{Study methods}

A new mobile digital thoracic drainage system, ATMOS ${ }^{\circledast}$ S021 Thorax (ATMOS MedizinTechnik GmbH \& Co. KG, Lenzkirch, Germany), is suitable for being transported within a hospital and for managing a pneumothorax patient, and is characterized by fully electronic monitoring, automatic rinsing function, real-time flow measurement, flow history, data export via USB stick, warnings, night mode and ergonomic touchscreen color display. We evaluated the effectiveness of this drainage system in terms of intrathoracic pressure and air leak in real time. This research involved adult patients admitted to the Department of Thoracic and Cardiovascular Surgery and to the intensive care unit who were diagnosed with primary or secondary pneumothorax requiring chest drainage and management. The patients were managed with a digital device $\left(\right.$ ATMOS $^{\circledR}$ S021 Thorax) which allows continuous monitoring and suction of air leaks. Chest tube drains were removed when there were no air leaks and the lung fully expanded in radiologic imaging studies.

\section{Statistical analysis}

Statistical Analyses were performed using MedCalc for Windows version 17.11.5 (MedCalc software, Ostend, Belgium) and the IBM SPSS software (version 21; IBM Corp., Armonk, NY, USA). All data were collected and analyzed using Microsoft Excel (Microsoft, Redmond, WA, USA). Continuous variables were tested for normality using the Kolmogorov-Smirnov test. Continuous variables showing normality were analyzed using Student's $t$ test and are expressed as the arithmetic mean \pm standard deviation, and those not showing normality were analyzed using the Mann-Whitney $U$ test and are expressed as the median (25-75th interquartile range). Categorical variables are displayed as frequency distributions and were evaluated with Pearson's Chi-square test or Fisher's exact test. Univariate comparisons between the groups for categorical variables were made using the Chi-square test and Fisher's exact test as appropriate. To avoid type 1 errors, Bonferroni post hoc correction (B-corrected) was applied to data that were initially deemed statistically significant by multiplying the number of variables by the $P$ value. Cox proportional hazards model was used to determine independent predictors of successful weaning. Overall survival was calculated according to the Kaplan-Meier method. Independent predictors of overall survival were also determined by using the Cox proportional hazards model. Statistical significance was set at $\mathrm{P}<0.05$. To identify independent factors associated with patient death, we used univariate and multivariate stepwise logistic regression models. Multiple logistic regression analysis using backwards stepwise regression was performed. Variables with a level of significance defined as $\mathrm{P}<0.20$ for univariate logistic regression analysis, as well as clinically important variables, were analyzed as independent predictors for the multivariate models. The data are expressed as odds ratios (OR) with $95 \%$ confidence intervals (CI) and relevant $\mathrm{P}$ values. To assess the predictive power of the logistic regression model, receiver operating characteristic (ROC) curves were used, and we calculated the area under the curve (AUC). The Hosmer-Lemeshow goodness-of-fit test was used to compare the numbers of observed and predicted deaths in risk groups for the entire range of 
death probabilities. Discrimination was assessed using the area under the ROC curves. Cumulative survival curves as a function of time were generated by the Kaplan-Meier approach and were compared between the groups using the log rank test.

\section{Institution approval}

The study was conducted in accordance with the Declaration of Helsinki (as revised in 2013).

This study was approved by the Institutional Ethics Committee/Review Board of Konkuk University Chungju Hospital. Informed consent was waived due to its retrospective nature of this study (IRB approval No., KUCH 2019-10-031).

\section{Results}

Among 240 patients treated with chest tubes in our institution between 2017 and 2018, we identified 100 eligible for analysis according to the above-mentioned criteria. The detailed demographic and clinical characteristics of the study patients are summarized in Table 1. Among the 100 patients, 50 were allocated to the digital group (male/female $=46 / 4$ ) and 50 patients were allocated to the analogue group (male/female $=45 / 5$ ). The baseline parameters in the digital and analogue groups were comparable with no significant differences in hospital length of stay (days) $(17.64 \pm 12.23$ vs. $18.32 \pm 16.64$, $\mathrm{P}=0.902)$, age $(52.92 \pm 21.17$ vs. $54.26 \pm 23.26, \mathrm{P}=0.764)$, height $(168.16 \pm 7.86$ vs. $167.16 \pm 12.62 ; \mathrm{P}=0.637)$, weight $(59.55 \pm 10.80$ vs. $59.53 \pm 11.10 ; \mathrm{P}=0.993)$, and body mass index $(21.06 \pm 3.66$ vs. $21.48 \pm 4.41, \mathrm{P}=0.605)$. There was no significant difference between the 2 groups in sex, smoking history, smoking period, underlying COPD, cardiac disease, diabetes mellitus or hypertension. There was no significant difference between the 2 groups in baseline characteristics, including pneumothorax site, pneumothorax size, pneumothorax severity, tension pneumothorax, first episode or recurrence, additional need for chest tube, admission route, surgical approach, type of surgical procedure or recurrence after discharge. Within the entire patient cohort, there was no mortality. In the analogue group, only 3 cases of chest tube clogging and/or kinking, which caused chest tube malfunction and/or blockage of tube drain, were identified as major complications. Four cases of chest tube site dehiscence requiring re-suture and 2 cases of residual pneumothorax after chest tube removal were treated with conservative treatment and were classified as minor complications. The mean hospital length of stay (days) was $18.14 \pm 14.53$, which was shorter in the digital group than in the analogue group, but there was no statistically significant difference in hospital length of stay between the 2 groups $(17.96 \pm 12.23$ vs. $18.32 \pm 16.64, \mathrm{P}=0.902)$. Air leakage time through a closed thoracostomy tube (hours) or indwelling time of a closed thoracostomy tube (hours) was not significantly different between the digital and analogue groups $(213.47 \pm 219.80$ vs. $261.94 \pm 184.47$, $\mathrm{P}=0.235$ and $223.44 \pm 218.75$ vs. $275.29 \pm 186.06, \mathrm{P}=0.205$, respectively) (Figure 1). The mean total drainage amount was $186.05 \pm 115.81 \mathrm{~mL}$, and the drainage amount was significantly greater in the digital group than in the analogue group $(209.62 \pm 139.63$ vs. $162.48 \pm 80.42, \mathrm{P}=0.042)$. Ambulation time per day was significantly different between the digital and analogue groups $(6.42 \pm 3.62$; SE, $0.51 ; 95 \%$ CI of difference, 5.39-7.44 vs. 3.94 \pm 1.74 ; SE, 0.24; $95 \%$ CI of difference, 3.44-4.43; $\mathrm{P}<0.001)$. Time to full expansion (hours) was significantly different between the digital and analogue groups $(25.64 \pm 14.55$; SE, 20.5; 95\% CI of difference, 21.50-29.77 vs. 46.52 \pm 25.53 ; SE, 3.61; 95\% CI of difference, 39.26-53.77; $\mathrm{P}<0.001)$. Sleep disturbance by closed thoracotomy tube drainage noise was significantly different between in the digital and analogue groups $(2.38 \pm 1.03 ;$ SE, $0.14 ; 95 \%$ CI of difference, 2.08-2.67 vs. 5.70 \pm 2.87 ; SE, 0.40 ; 95\% CI of difference, 4.88-6.51; $\mathrm{P}<0.001$ ) (Table 2).

\section{Discussion}

The concept of thoracic drainage using a bottle system was widely used after the 1918 influenza epidemic to drain post-influenza empyema. In 1926, Lilienthal employed a pair of thoracic bottles for the postoperative management of pulmonary resection for bronchiectasis. In this twocompartment system, the one, a so-called collection bottle, which was connected to the thoracic drainage tube, collected pleural fluid, and the other, a so-called rigid tube with a water-sealed bottle which submerged under $2.0 \mathrm{~cm}$ of water, drained air from the pleural space. The two-compartment bottle system showed a great strength in the ability to drain a larger quantities of fluid, such as blood, pus, chyle and fluid, from the pleural cavity without any decrease in the efficiency of drainage which frequently occurred in the one-compartment device. In 1952, Howe introduced the three-compartment system consisting of a collection bottle, a water-seal bottle and a suction 
Table 1 Demographic characteristics of the enrolled patients.

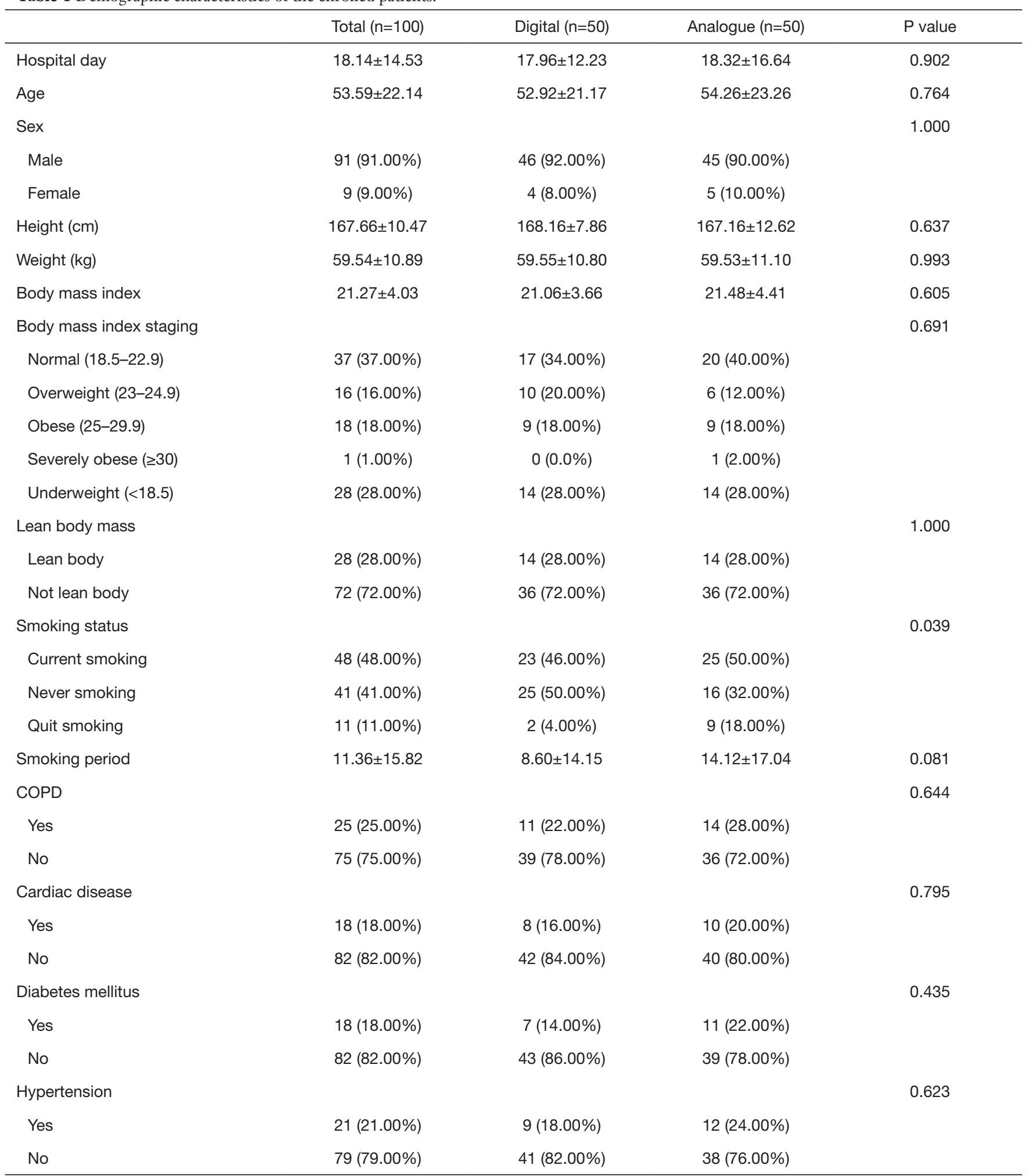

Table 1 (continued) 
Table 1 (continued)

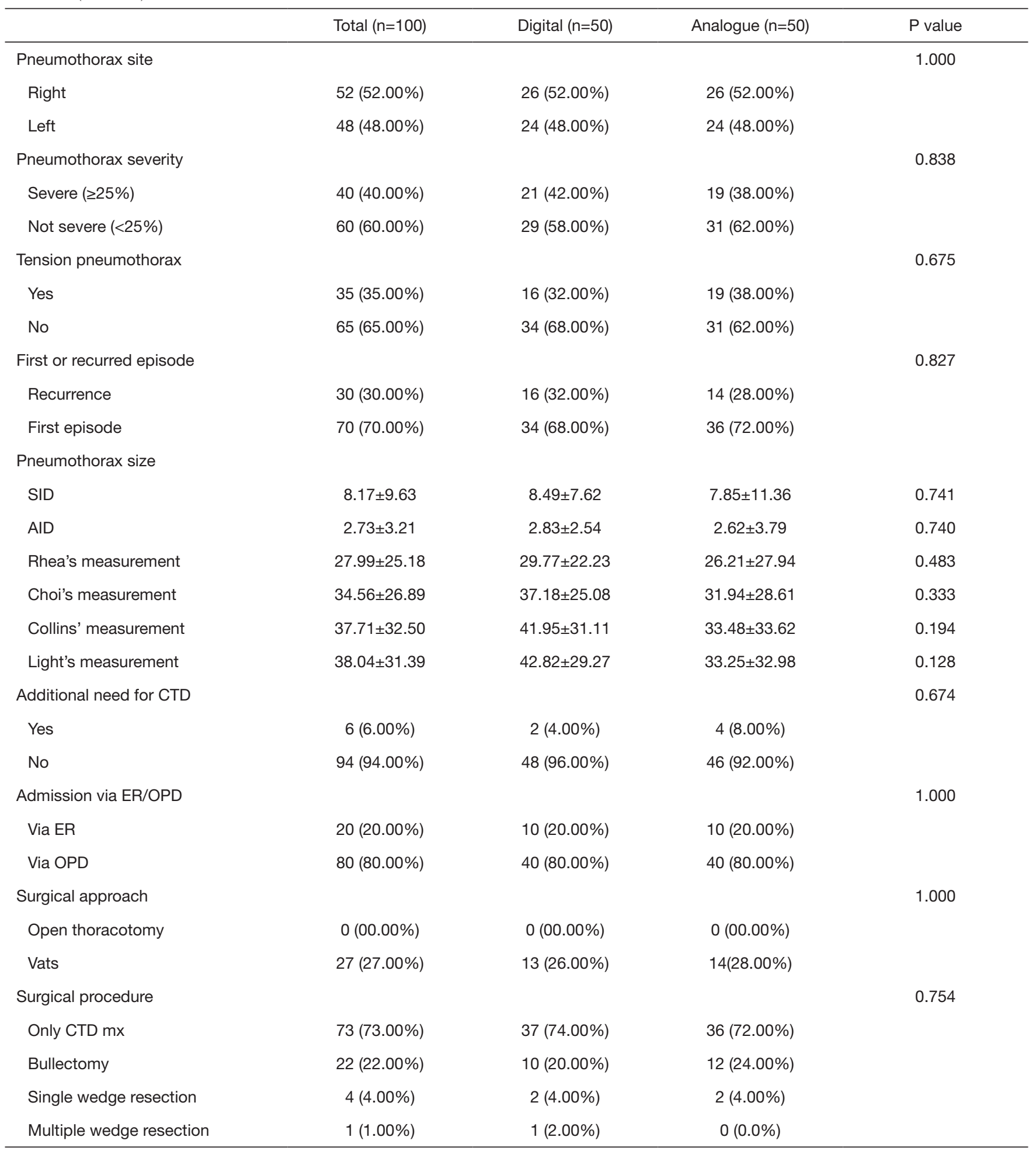

Table 1 (continued) 
Table 1 (continued)

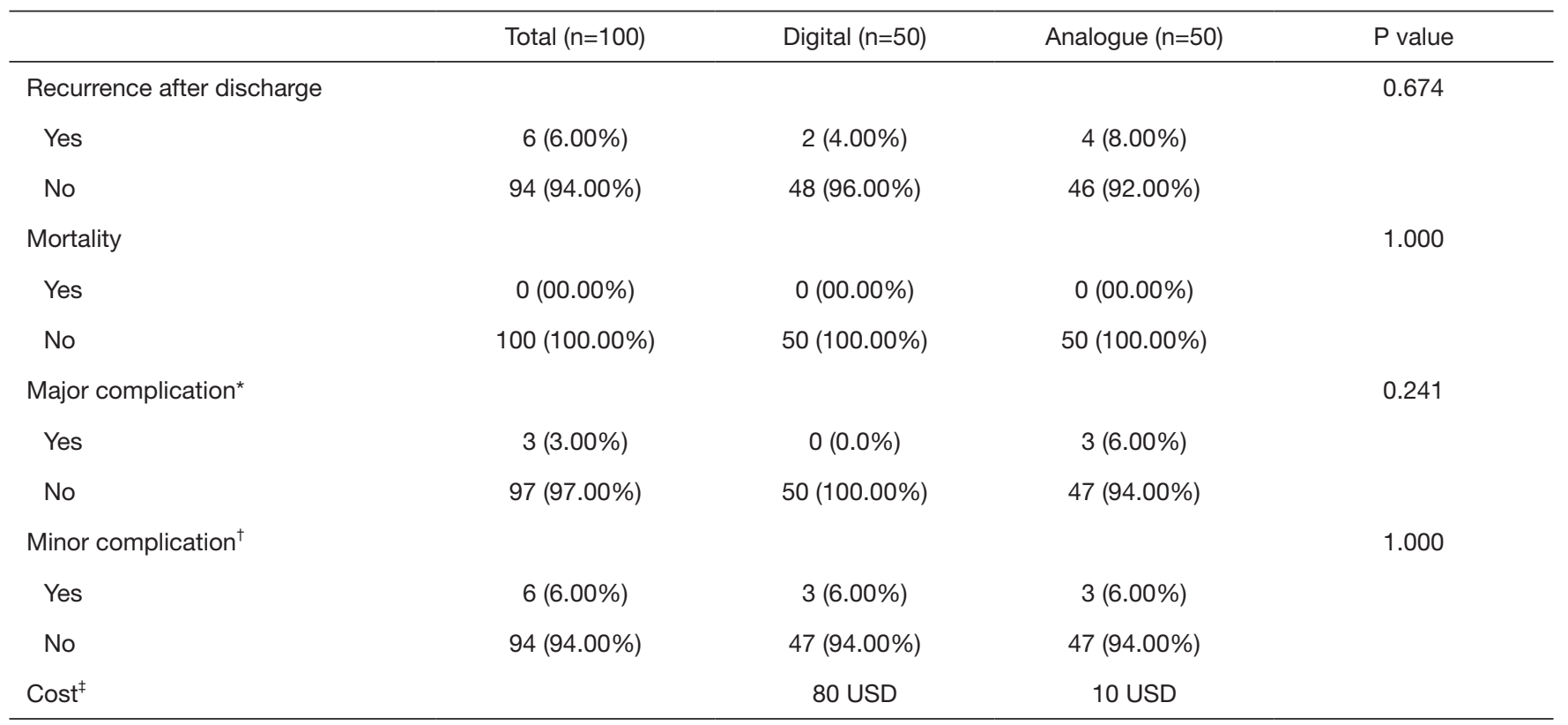

Data are presented as the arithmetic mean \pm standard deviation. *Major complications include 3 cases of chest tube malfunction and/or blockage of the chest tube. ${ }^{\dagger}$ Minor complications include 4 cases of chest tube site dehiscence requiring re-suture and 2 cases of residual pneumothorax after chest tube removal. ${ }^{\ddagger}$ The costs of chest bottle in both the digital and analogue groups were fully covered by national health insurance service. The Korean won to US dollar exchange rate was 1,200 Korean won: 1 US dollar. COPD, chronic obstruction pulmonary disease; SID, sum of interpleural distance on pneumothorax; AID, average of interpleural distance on pneumothorax; CTD, closed thoracostomy drainage; ER, emergency room; OPD, out-patient department; P, statistical probability.

control (manometer) bottle, which provided the basis for contemporary thoracic drainage devices. The pleural drain from a patient is connected to the collection bottle as a first bottle and serves as a drainage compartment; the collection bottle is connected to the middle compartment as a second bottle and serves as the underwater-seal bottle; and the underwater-seal bottle is connected to the third compartment for the control of negative pressure based on the depth of rigid tube submersion below the fluid surface. The bottles of the three-compartment system are connected in series to the thoracic drainage tube in this way. The three-chamber system first designed by Dr. Howe precisely enables physicians to evaluate the proper patency of a chest tube, to confirm the intrapleural location of the chest tube through interpretation of fluid column oscillation corresponding to changes in intrapleural pressure, also known as the tidaling phenomenon, to identify air leakage presenting as a bubbling sign within the water-seal chamber, and to subjectively quantify air leakage volume. Based on the closed water seal drainage system, chest tube management became the standard of care in the early 20th century, a major turning point in the history of thoracic surgery. The chest tube had regularly been used to drain the chest cavity after thoracotomy during World War II and had routinely been used for emergency tube thoracostomy following acute trauma until the Korean War; consequently, postoperative chest tube placement is currently the gold standard procedure after major/minor thoracic surgery. With recent advances in medical technology, some studies showed positive results on conversion of chest drainage from analogue to digital suction systems.

Complications by chest tube placement include primary and secondary injuries to the lungs, intercostal/intrathoracic vasculature, esophagus, stomach, liver, spleen, diaphragm, major blood vessels and even cardiac structures. In very extremely rare cases, improper placing of a chest tube far into the thorax results in perforation of the heart, injuries to large vessels, perforation of the esophagus and nerve injuries, which in turn leads to fatal consequences such as massive bleeding, organ injury/failure and mortality. These complications can be categorized as tube malposition (i.e., intraparenchymal, fissural, chest wall, mediastinal and/or abdominal placement), blockage of tube drainage (which may be due to kinking, angulation, clot formation within 
A

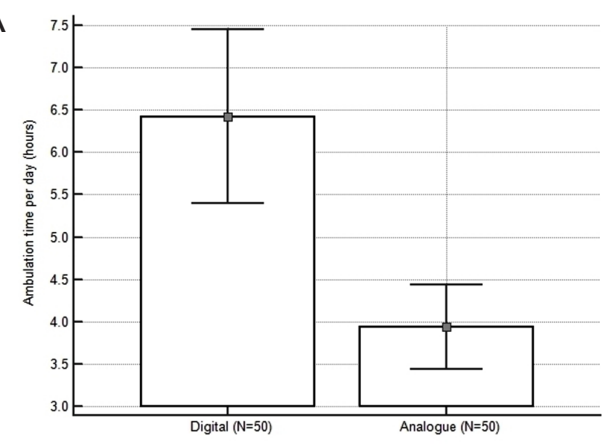

C

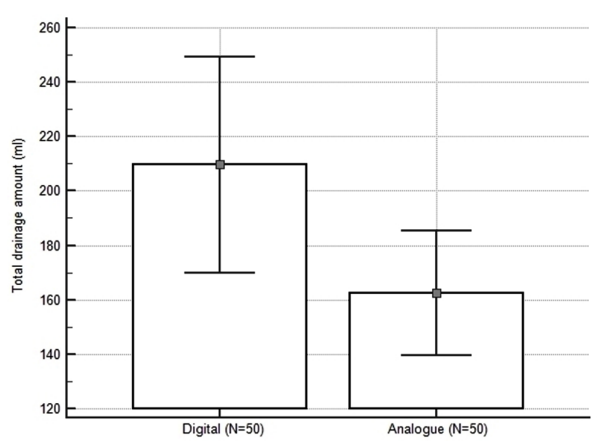

E

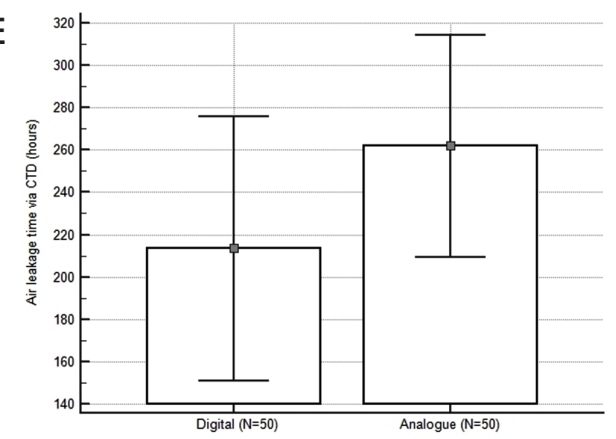

G

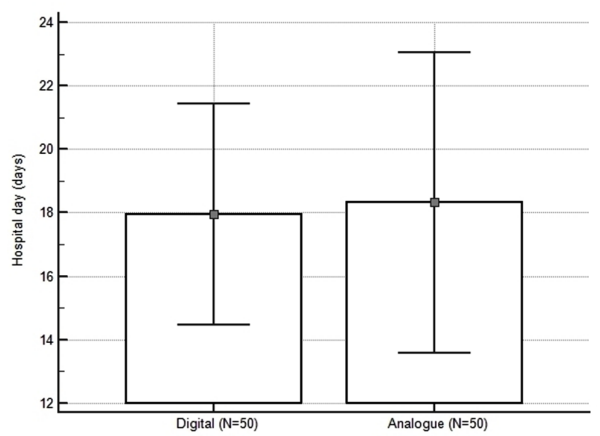

B

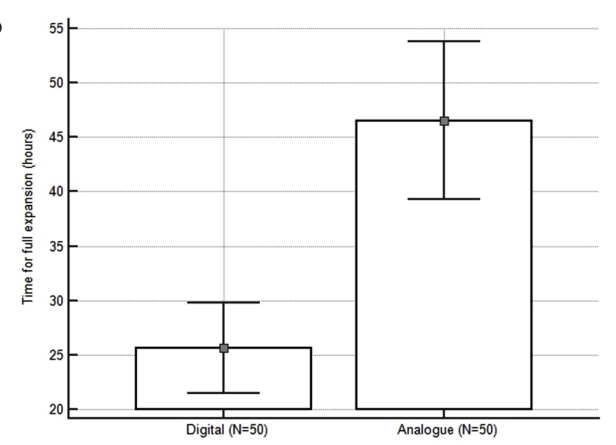

D

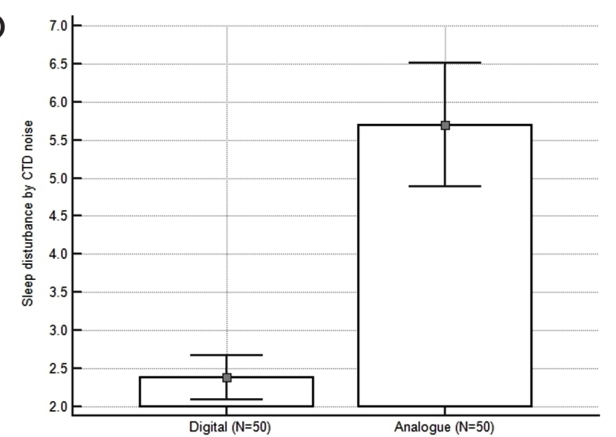

F

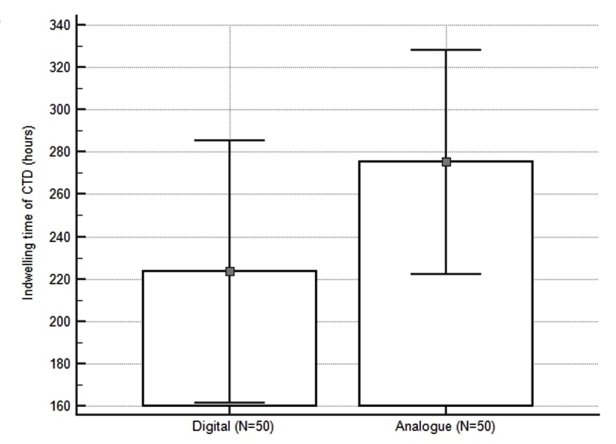

$\mathrm{H}$

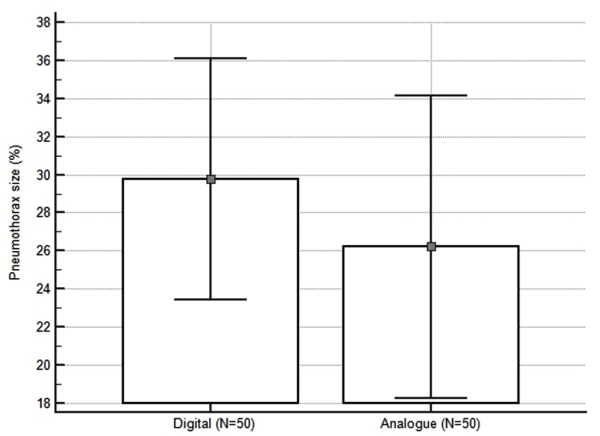

Figure 1 Comparison between the digital and analogue groups. The columns and dots show group mean values with standard error bars. More detailed data are summarized in Tables 1 and 2. (A) Ambulation time each day (hours): $\mathrm{AMD}=2.48 ; \mathrm{SD}=2.83 ; \mathrm{SE}=0.56 ; \mathrm{P}<0.0001$. (B) Time to full expansion (hours): $\mathrm{AMD}=20.87 ; \mathrm{SD}=20.77 ; \mathrm{SE}=4.15 ; \mathrm{P}<0.0001$. (C) Total drainage amount (ml): $\mathrm{AMD}=47.14 ; \mathrm{SD}=113.93$; $\mathrm{SE}=22.78 ; \mathrm{P}=0.0412$. (D) Sleep disturbance by $\mathrm{CTD}$ noise: $\mathrm{AMD}=3.31 ; \mathrm{SD}=2.15 ; \mathrm{SE}=0.43 ; \mathrm{P}<0.0001$. (E) Time to air leakage via CTD (hours): $\mathrm{AMD}=48.46 ; \mathrm{SD}=205.96 ; \mathrm{SE}=40.58 ; \mathrm{P}=0.2352$. (F) Time for indwelling $\mathrm{CTD}$ (hours): $\mathrm{AMD}=51.85 ; \mathrm{SD}=203.06 ; \mathrm{SE}=40.61$; $\mathrm{P}=0.2047$. (G) Hospital day (days): $\mathrm{AMD}=0.36 ; \mathrm{SD}=14.60 ; \mathrm{SE}=2.92 ; \mathrm{P}=0.9022$. $(\mathrm{H})$ Pneumothorax size (\%): $\mathrm{AMD}=3.55 ; \mathrm{SD}=25.24$; $\mathrm{SE}=5.04 ; \mathrm{P}=0.4831$. AMD, arithmetic mean difference; $\mathrm{SD}$, standard deviation; SE, standard error; $\mathrm{P}$, statistical probability; CTD, closed thoracostomy drainage. 
Table 2 Major statistical analyses between the digital and analogue groups

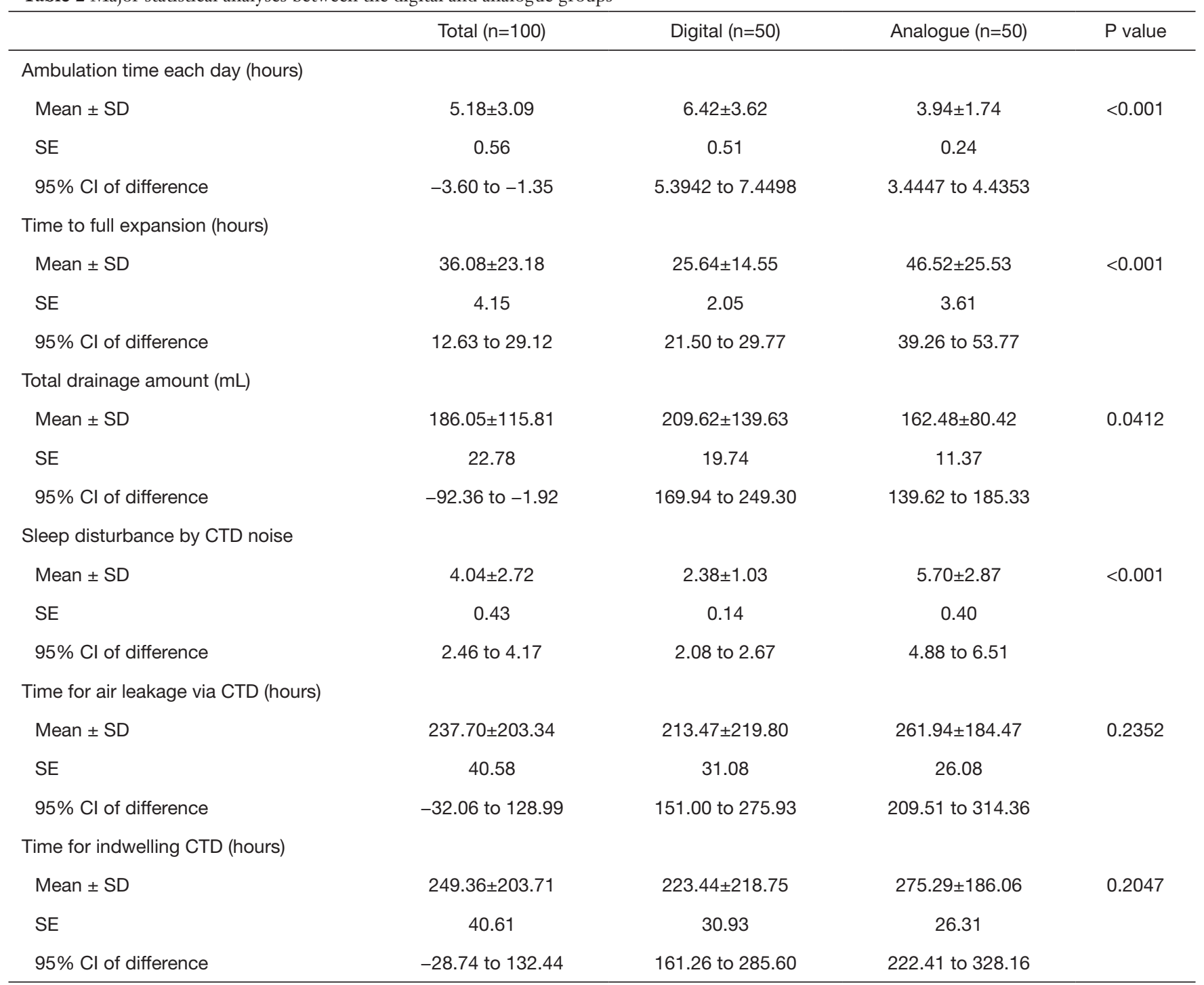

Data are presented as the arithmetic mean \pm standard deviation. SD, standard deviation; SE, standard error; $\mathrm{Cl}$, confidence interval; $\mathrm{P}$, statistical probability.

the lumen, or the presence of debris or lung tissue), chest drain dislodgement, re-expansion pulmonary edema, subcutaneous emphysema, nerve injury (i.e., Horner's syndrome, phrenic nerve injury and injury to the long thoracic nerve), cardiac/vascular injury (i.e., cardiac injury, injury to the pulmonary artery, occlusion of the subclavian artery, intercostal artery injury and/or chest wall arteriovenous fistula), esophageal perforation, bronchocutaneous fistula, cardiac dysrhythmia, herniation of a lung bulla through the insertion site, infection complications (i.e., empyema and tube site infection), wound complications (i.e., dehiscence requiring secondary closure) and/or residual/post-extubation pneumothorax (1-5). In the case of chest tube, persistent air leak into the pleural space is a very frustrating challenge to chest and thoracic surgeons, which mostly develops in association with bronchopleural or alveolopleural fistulas. These fistulas frequently occur following pulmonary resection and along with primary or secondary pneumothorax. The purpose of chest tube drainage is based on successful elimination of air, fluid, pus, chyle and blood from the pleural space, resulting in rapid lung expansion and restoration of pulmonary function. Persistent and prolonged air leak is one of the most frequent complications after cardiothoracic surgery, which 
can suppress re-expansion of the lungs, prolong hospital stays and increase hospital costs. Successful management and early return to daily life activities in patients with pneumothorax depend on proper thoracic tube management and accurate assessment of the type and magnitude of air leaks. To date, assessment and grading of air leaks have been based on static analog measurement of the numbered column through which bubbling occurs in the collection chamber. This classic assessment mainly relies on clinicians' subjective interpretation and personal experience. This imprecise assessment leads to incorrect decisions about maintenance of chest tube drainage as well as the need for pleurodesis or surgical repair $(6,12)$. Discrepancies exist in patient care such as evaluation of the size of an air leak though a chest tube and the absence or presence of an air leak, despite technological advancement, verified analysis and application of the same air leak classification system (12).

In previous reports, the air leak has been described as expiratory, inspiratory, continuous or forced expiratory, while the number of chambers on the meter demonstrating bubbles have quantified the degree of air leakage. It is noteworthy that suction might increase the air leak volume, contribute to discomfort from the chest tube under negative intrapleural pressure and to prolong hospitalization due to maintenance of the alveolo-pleural fistula. Constant bubbling in the third compartment during suction can also cause patient distress due to noise. Therefore, appropriate evaluation of chest tube function and proper maintenance of chest tube suction would be an important part of thoracic treatment. Recently, new pleural drainage devices have been manufactured and commercially available, which allow for pleural pressure assessment via digital quantification of air leak through a chest tube. It is considered that these new systems would enable objective and consistent assessment of an air leak by quantifying subjective bedside clinical parameters and could allow for earlier mobilization and ambulation through unique characteristics of portable systems: no need for connecting to a wall-mounted suction system and less difficulty in manipulating the new suction system. Earlier devices, such as Digivent (Millicore A.B., Sweden) and Heimlich valve (Airfix, University of Technology, Graz, Sweden), are currently not commercially available. At present, ATMOS ${ }^{\circledR}$ (Atmosmed, Allentown, PA, USA) and Thopaz (Medela, Inc, Baar, Switzerland) are commercially available pleural drainage devices, which measure pleural pressure and flow rate in real time through built-in screens and digital sensors. The measured data are saved and displayed on a built-in screen as trends, and can be downloaded for additional examination and analysis (Figures 2 and 3). These devices also enable chest surgeons to accurately examine what changes occur in the pleural space in patients with pneumothorax during both medical and surgical treatments. Theoretically, objective assessment of air leak has advantages over subjective assessment in that the former provides bubble counts in a chest tube to determine the optimal timing of chest tube removal. Recently, chest drainage devices with stand-alone suction pumps have been developed and commercially available. Because these new-type devices do not constrain patients to a wall suction device, patients can ambulate earlier in the surgical ward under proper active suction. However, it is still debatable whether application of suction would be helpful in all patients with chest tubes and, if so, how much the suction pressure should be applied. The debate may arise from different surgical procedures and clinical features of patients with pneumothorax. A previous study has demonstrated that the passive suction or gravity mode is sufficient to treat most patients with pneumothorax and to reduce the length of air leak (3-6). Another study has indicated that dual modalities of active suction at night and patient ambulation in the daytime are sufficient to treat patients with pneumothorax (3-6). Portable pumps that can control intrapleural pressure provide more information on clinical conditions of patients compared to active suction devices that cannot control intrapleural pressure. A recent study reported that digital thoracic drainage systems reduced differential intrapleural pressure in patients undergoing upper lobectomy, whereas they did not in those undergoing lower lobectomy (13-16). Although monitoring with these digital systems provides objective data on intrapleural space abnormalities, further studies are needed to confirm these results (17). Notably, some differences in suction pressure or negative pressure may be present in a chest tube when active suction is not applied. Therefore, chest tube drainage using suction or water seal is regarded as passive drainage, and this passive suction may be affected by various factors, including the difference in the height of the system and the chest tube tip inside the thoracic cavity. The suction pressure is usually reported to be $5-8 \mathrm{cmH}_{2} \mathrm{O}$. Portable digital thoracic drainage systems might control intrapleural pressure, lead to more stable conditions, and thus help restore pulmonary function (18).

Despite the authors' short knowledge and limited experience, digital thoracic drainage systems offer some advantages over traditional analogue systems. In patients managed with chest tube drainage, digital thoracic drainage 


\begin{tabular}{|c|c|c|c|c|c|}
\hline \multirow{2}{*}{$\mathrm{A}_{1}$} & A & B & c & D & E \\
\hline & THERAPY REPORT & & & & \\
\hline \multicolumn{6}{|l|}{2} \\
\hline 3 & Patient: & & & & \\
\hline 4 & Diagnosis: & & & & \\
\hline 5 & Secretion: & & & & \\
\hline 6 & Air leak - Flow @ Start: & $0.00 \mathrm{l} / \mathrm{min}$ & & & \\
\hline 7 & Air leak - Flow @ End: & $0.00 \mathrm{l} / \mathrm{min}$ & & & \\
\hline \multicolumn{6}{|l|}{8} \\
\hline 9 & Start date: & 09.01 .2018 / 12:31 & & & \\
\hline 10 & End date: & 09.12.2018 / 12:31 & & & \\
\hline 11 & Recording time: & 12:00:00:00 & & & \\
\hline \multicolumn{6}{|l|}{12} \\
\hline 13 & Creation date of report: & :09.14.2018 / 12:32:59 & & & \\
\hline \multicolumn{6}{|l|}{14} \\
\hline 15 & Device name: & ATMOS?S 201 Thorax & & & \\
\hline 16 & Device ID: & $00 \mathrm{C} 2$ & & & \\
\hline 17 & PCB hardware version: & V1.0.1 & & & \\
\hline 18 & Firmware version: & V3.0.40 & & & \\
\hline \multicolumn{6}{|l|}{19} \\
\hline 20 & $\#$ & TIME & TARGET VACUUM & MEASURED VACUUM & FLOW \\
\hline 21 & & [dd:hh:mm:ss] & [mbar] & [mbar] & {$[\mathrm{ml} / \mathrm{min}]$} \\
\hline 22 & & $00: 00: 00: 00$ & -20 & -20 & 0 \\
\hline 23 & & $00: 00: 06: 00$ & -20 & -20 & 0 \\
\hline 24 & & $00: 00: 12: 00$ & -20 & -20 & 0 \\
\hline 25 & & $00: 00: 18: 00$ & -20 & -17 & 0 \\
\hline 26 & & $00: 00: 24: 00$ & -20 & -20 & 0 \\
\hline 27 & & $00: 00: 30: 00$ & -20 & -20 & 0 \\
\hline 28 & & $00: 00: 36: 00$ & -20 & -20 & 0 \\
\hline 29 & & $00: 00: 42: 00$ & -20 & -20 & 0 \\
\hline 30 & & $00: 00: 48: 00$ & -20 & -18.3 & 0 \\
\hline 31 & & $00: 00: 54: 00$ & -20 & -19 & 0 \\
\hline 32 & & 00:01:00:00 & -20 & -20 & 0 \\
\hline 33 & & $00: 01: 06: 00$ & -20 & -20 & 0 \\
\hline 34 & & $00: 01: 12: 00$ & -20 & -20 & 0 \\
\hline 35 & & 00:01:18:00 & -20 & -20 & 0 \\
\hline 36 & & $00: 01: 24: 00$ & -20 & -20 & 0 \\
\hline 37 & & $00: 01: 30: 00$ & -20 & -20 & 0 \\
\hline 38 & & $00: 01: 36: 00$ & -20 & -20 & 0 \\
\hline 39 & & $00: 01: 42: 00$ & -20 & -19 & 0 \\
\hline 40 & & $00: 01: 48: 00$ & -20 & -20 & 0 \\
\hline 41 & & $00: 01: 54: 00$ & -20 & -20 & 0 \\
\hline 42 & & $00: 02: 00: 00$ & -20 & -20 & 0 \\
\hline 43 & & $00: 02: 06: 00$ & -20 & -19 & 0 \\
\hline 44 & & $00: 02: 12: 00$ & -20 & -20 & 0 \\
\hline 45 & & $00: 02: 18: 00$ & -20 & -19.5 & 0 \\
\hline 46 & & $00: 02: 24: 00$ & -20 & -20 & 0 \\
\hline 47 & & $00: 02: 30: 00$ & -20 & -20 & 0 \\
\hline 48 & & $00: 02: 36: 00$ & -20 & -21 & 0 \\
\hline 49 & & $00: 02: 42: 00$ & -20 & -16.3 & 0 \\
\hline 50 & & $00: 02: 48: 00$ & -20 & -19.8 & 0 \\
\hline 51 & & $00: 02: 54: 00$ & -20 & -20 & 0 \\
\hline 52 & & $00: 03: 00: 00$ & -20 & -22.3 & 0 \\
\hline 53 & & $00: 03: 06: 00$ & -20 & -20 & 0 \\
\hline 54 & & $00: 03: 12: 00$ & -20 & -20 & 0 \\
\hline 55 & & $00: 03: 18: 00$ & -20 & -20 & 0 \\
\hline 56 & & $00: 03: 24: 00$ & -20 & -20 & 0 \\
\hline 57 & & $00: 03: 30: 00$ & -20 & -20 & 0 \\
\hline 58 & & $00: 03: 36: 00$ & -20 & -20 & 0 \\
\hline 59 & & $00: 03: 42: 00$ & -20 & -20 & 0 \\
\hline 60 & & $00: 03: 48: 00$ & -20 & -20 & 0 \\
\hline 61 & & 00:03:54:00 & -20 & -20 & 0 \\
\hline 62 & & $00: 04: 00: 00$ & -20 & -20 & 0 \\
\hline 63 & & $00: 04: 06: 00$ & -20 & -20 & 0 \\
\hline 64 & & $00: 04: 12: 00$ & -20 & -19 & 0 \\
\hline 65 & & $00: 04: 18: 00$ & -20 & -20 & 0 \\
\hline 66 & & $00: 04: 24: 00$ & -20 & -20.3 & 0 \\
\hline 67 & & $00: 04: 30: 00$ & -20 & -20 & 0 \\
\hline 68 & & $00: 04: 36: 00$ & -20 & -21.5 & 0 \\
\hline 69 & & $00: 04: 42: 00$ & -20 & -20 & 0 \\
\hline 70 & & $00: 04: 48: 00$ & -20 & -22.5 & 0 \\
\hline 71 & & $00: 04: 54: 00$ & -20 & -20 & 0 \\
\hline 72 & & $00: 05: 00: 00$ & -20 & -20 & 0 \\
\hline 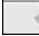 & ATMOS_S_20 & 01_THORAX_IDOOC2_2020. & $\oplus$ & $\vdots 1$ & \\
\hline
\end{tabular}




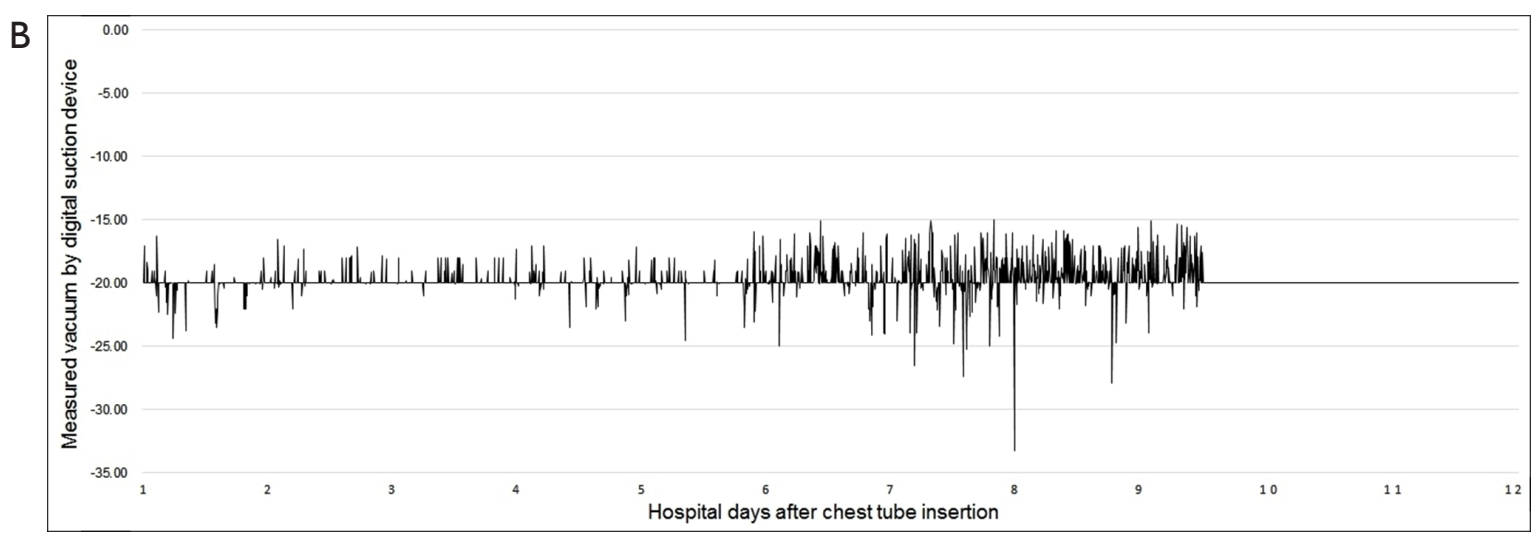

Figure 2 (A) Representative data from the digital suction device. This figure shows target vacuum (mbar), measured vacuum (mbar) and flow $(\mathrm{mL} / \mathrm{min})$, based on data extracted from a case of a 17 -year-old male patient with spontaneous pneumothorax who was treated only with closed thoracostomy drainage using a digital suction device without any surgical approaches. (B) Measured vacuum depicted in the same patient data. The fluctuations in vacuum were diminished and stabilized at the end of the ninth hospital day. This implies that the air leakage is completely ceased, indicating the optimal timing of chest tube removal.

systems have advantages in terms of morbidity, ambulation and exercise, early return to everyday activities, shortening of recovery time and improvement in quality of life. All of the digital thoracic drainage systems consisting of transparent canisters provide the consistency and color of fluid/secretion for clear vision, easy balancing and hygienic disposal. Furthermore, with the intuitive help of real-time data collection and vacuum measurement, patients can be supported by adaptation of individually regulated vacuum, objective evaluation of thoracic pressure at a glance and more customized treatment $(15,19,20)$. With the support of the digital technique, automatic regular checkups are always available and reduce work load of physicians and nursing staff, especially during night and at weekends (16). The most important benefit from the digital thoracic drainage system would be early detection of chest tube clogging frequently causing tension pneumothorax and/or cardiac tamponade, which prevents critical and fatal complication and improves treatment outcome. Although there were no significant differences between the digital thoracic and traditional analogue suction systems, the digital thoracic drainage system had shorter hospitalization days and indwelling time for chest tube drainage $(15,16,19-21)$. After application of the digital suction system, the authors were able to find several considerations, improvement points and limitations compared to the traditional analogue system. Since the digital suction system has no rubber connection between a chest tube and a canister which enables squeezing or milking for better drainage, it has a repetitive mechanical auto-rinsing system instead. Unfortunately, this new technology is not familiar to physicians and causes difficulty in assessing appropriate drainage and chest tube function. In our study, the drainage amount was larger and the time required for full expansion was shorter in the digital suction system than in the closed suction system $(209.62 \pm 139.63$ vs. $162.48 \pm 80.42$ and $25.64 \pm 14.55$ vs. $46.52 \pm 25.53$, respectively), which indicates sufficient squeezing or milking in the digital suction system. These results show that digital suction systems perform adequate drainage control, which regulate the vacuum on the patient's side, not on the device side, but conventional systems regulate only the vacuum on the device side, not on the patient's side. These results also indicate that digital suction systems have no siphon effect due to precise vacuum measurement and automatic hose rinsing function on the patient's side. In digital suction systems that adopt a free of or optional water sealing technology, evaluation of air leakage severity by checking air bubbling is not possible and chest tube clamping test is not effective in determining the optimal timing of chest tube removal, unlike traditional analogue suction systems. Because the digital suction system is designed based on a multi-chamber system and has one canister equipped with a first collection vacuometer and an optional water-seal chamber, much effort and time are required for physicians who are familiar with traditional analogue chest tube system. The digital suction device used in our study has a standardized hose system between a main digital machine and a chest tube, which minimizes the necessity for direct 


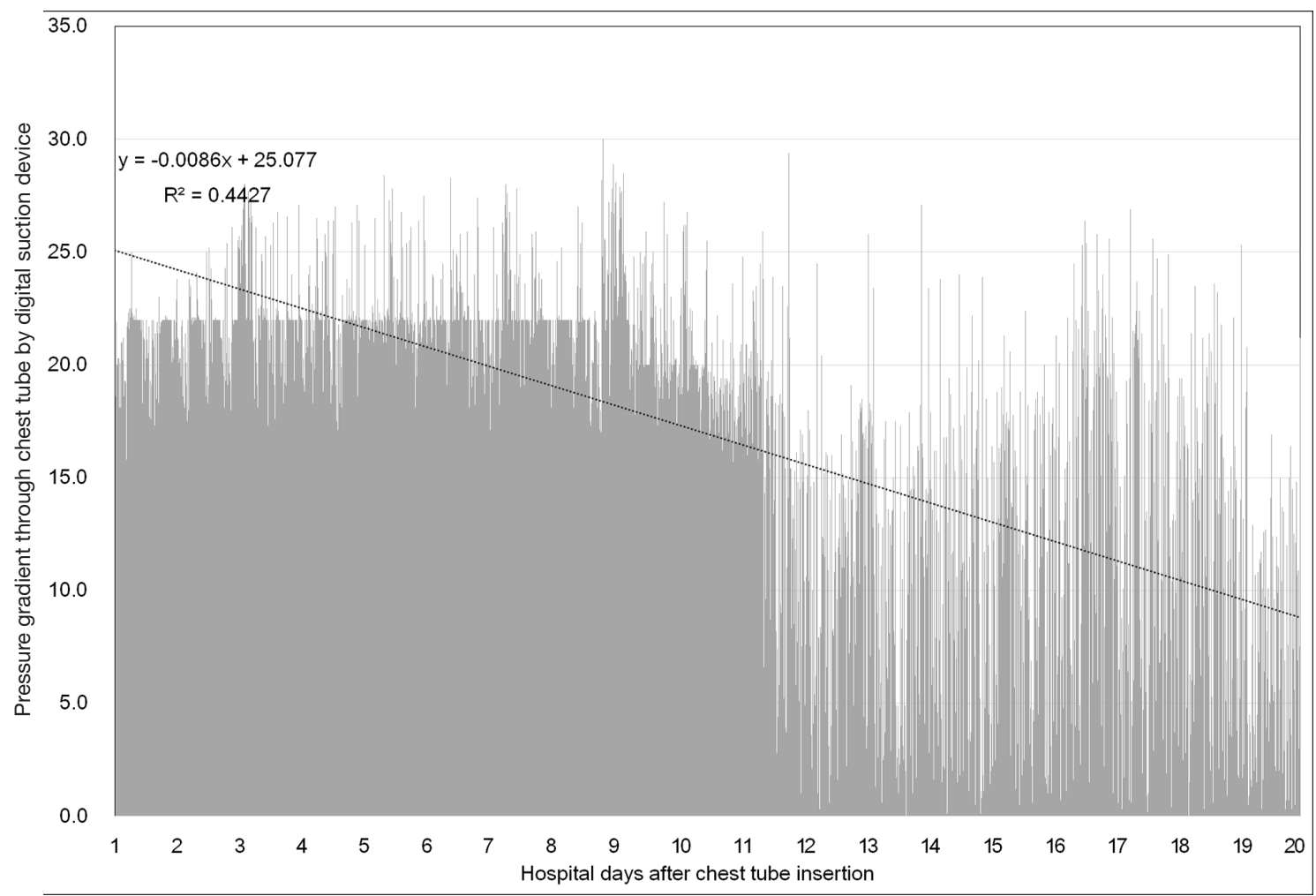

Figure 3 Histogram of pressure gradients through the chest tube. This figure shows pressure variations through the chest tube, based on data extracted from a case of a 67-year-old male patient with secondary pneumothorax who was treated only with closed thoracostomy drainage using a digital suction device without any surgical approaches. The pressure gradients were automatically checked every 6 seconds and saved at the server of the device. The saved data were easily identified through the integral on-board screen of the digital suction device and were efficiently extracted using a universal serial bus flash memory. The black dotted line indicates the trend of pressure gradients through the chest tube per hospital day $\left(y=-0.0086 x+25.077, \mathrm{R}^{2}=0.04427\right)$. Note that the pressure gradient sharply decreased approximately on the 11th hospital day, which indicates a reduction of mechanical demand on suction power for equilibrium. Clinically, it represents a decrease in pressure for the proper maintenance of intrathoracic pressure and means a reduction of air leakage from the injured pneumothorax lung. This figure was reconstructed and completed using Microsoft Excel (Microsoft, Redmond, WA, USA) on the basis of data from the digital suction device.

milking with the help of integrated hose rinsing and enables immediate sampling of secretions in the connector (Figure 4). However, in cases in which a small amount of fluid is collected, it is not completely removed and remains in the hose system after automatic hose rinsing, which occasionally makes it difficult to calculate actual fluid drainage. The 3-way stopcock for pleural fluid sampling located in the middle of the hose system becomes loose from its mount, causing sudden interruption of the device and ear-splitting alarm signals for rechecking the device and hose line. Air bubbling sign during automatic hose rinsing (about 2.0 seconds) makes physicians confuse it as a persistent air leak from the patient. The digital suction device used in our study consists of 5 chamber systems: a large-capacity canister system for the first 4 chambers is designed to collect 2 liters of fluid and a fifth chamber plays a role as a water seal bottle as in the analogue suction system. The water sealing is optional (dry $v s$. wet). In the case of water sealing, flow visualization through bubbles could be easily detected; the end of the air pathway of a chamber is equipped with a hydrophobic bacterial filter and plays an important role for integrated overflow protection and prevention of air back regurgitation. In several cases of optional water sealing, fluctuation of sealed water in the fifth chamber during ambulation or fall-down caused filter contamination, which made the device suction operation abruptly stop. Only replacement with a new filter could resolve this unexpected event. Intuitive device manipulation/ 

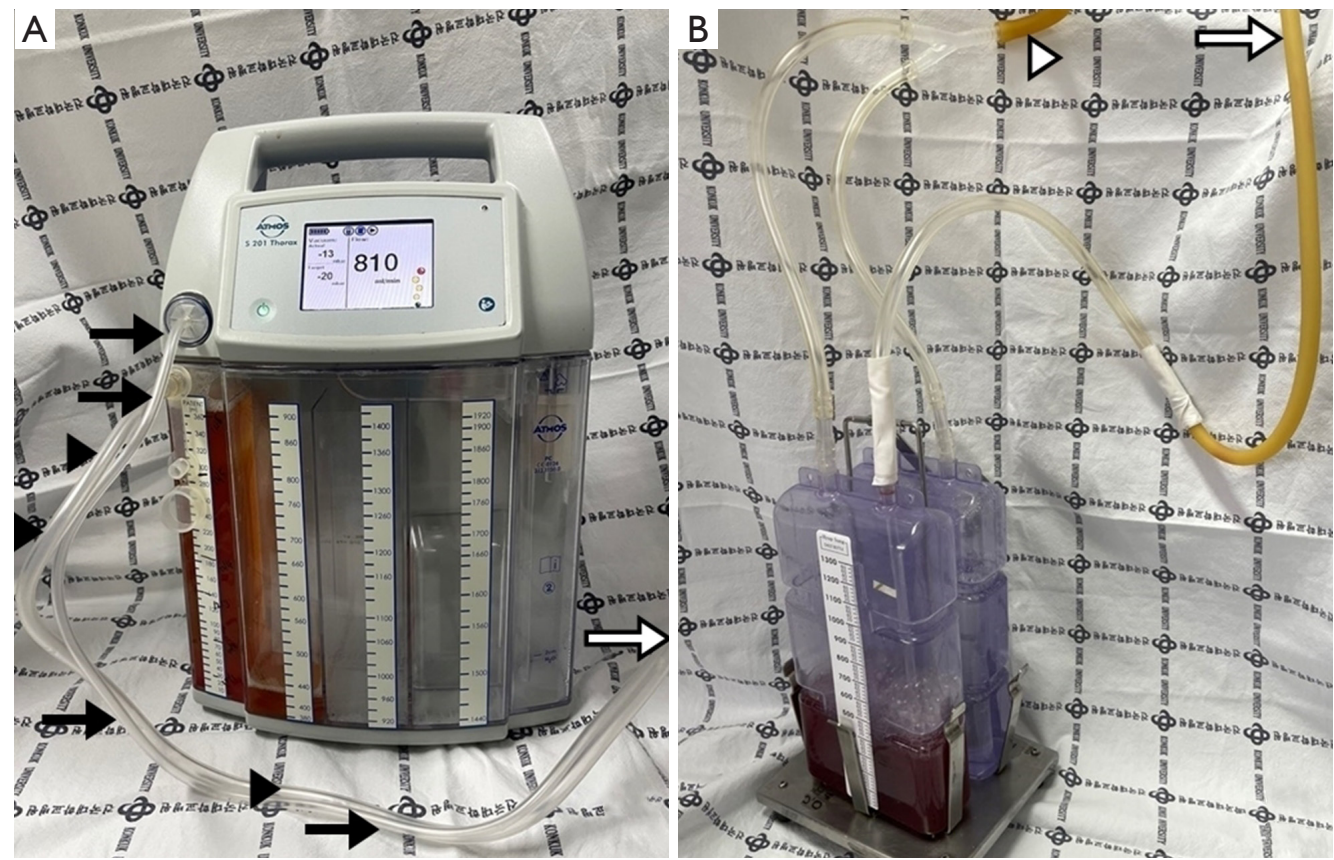

Figure 4 Comparison of view between the digital (A) and analogue (B) suction devices in the real clinical setting. The digital system uses a double-lumen hose system, which consists of a measuring/rinsing hose as well as a secretion hose. Black arrows indicate a measuring/ rinsing hose with an integrated hydrophobic bacterial filter, which prevents bacteria from entering the device. Black arrowheads indicate a secretion hose, through which secretions and air are suctioned and collected in a fully transparent secretion canister. The digital system has an automatic hose rinsing function, working periodically, 2 rinsing cycles every 3 minutes. The rinsing process transports secretions in the secretion hose to the secretion canister, which prevents accumulation of debris in the secretion hose, ingress of secretions into the measuring/rinsing hose, and creation of syphon effect. The automatic hose rinsing function enables sufficient drainage without milking and/or squeezing. The analogue suction device comprises 2 bottles, water sealed and suction control bottles. It has a thick yellowish rubber which is connected to the chest drainage tube for milking and/or squeezing and is mounted to the wall suction (empty arrowhead with black outline). The empty arrow with black outline indicates the tube connecting to the patient's chest tube in (A) and (B).

verification using a touchscreen provides user-friendly functionality for a convenient use of the device. Therapy data/information reading out through USB provides easy data storage. However, since the USB port is located to the posterior of the canister system, data export is never possible in the time of operation; thus, the only method for data extraction is turn-off the device and separation of the canister system. Both digital and analogue drainage systems are fully covered by national health care insurance (80 USD and 10 USD, respectively), and cost effectiveness and patient satisfaction are relevant to the shortened drainage time and the shorten length of hospital stay in the digital suction group (Table 1). Our study highlights the convenience and usefulness of the digital thoracic drainage system for the management of pneumothorax. Furthermore, the results of our study suggest that this system could be applied to patients, such as iatrogenic, catamenial and traumatic pneumothoraxes, hemothorax, chylothorax, pleural effusion and empyema.

This study has several limitations. First, this study was conducted at a single institution and male sex was predominant, which limited the generalizability of the study results. Secondly, our study population was relatively small and had a variety of underlying diseases indicated for closed thoracostomy drainage with a chest tube. Thirdly, since our study focused only on primary or secondary pneumothoraxes, it is difficult to generalize our results to other forms of pneumothorax, such as iatrogenic pneumothorax, catamenial pneumothorax or postoperative air leak. Further studies in patients with various forms of pneumothorax are warranted to see if our results can be applied to such patients. Fourthly, since this study concentrated only on patients with primary and secondary pneumothoraxes who were managed with simple surgical 
procedures, such as chest tube insertion, bullectomy and single/multiple wedge resection, it is difficult to generalize our results to other types of surgical procedures such as segmentectomy, lobectomy, bi-lobectomy and pneumonectomy, which are essential to post-operative air leak and chest tube drainage management. Fifthly, this study has some limitations stemming from its small sample size and retrospective design as well as its short-term followup. Further prospective multicenter studies are needed to confirm our results. Finally, there may have been errors in the measurement of definite pneumothorax amounts on posterior-anterior (PA) and/or anterior-posterior (AP) chest X-rays, but not on high-resolution computed tomography (HRCT). In several cases, furthermore, correct measurement of pneumothorax size was impossible because the inter-pleural distance was unable to be calculated due to partial collapse and/or adhesion (Figure S1 and Table S1).

Future research should develop a more simplified digital thoracic drainage device to accurately manage patients with pulmonary air leak and to explore the role of the device in clinical practice. In addition, multicenter, randomized, controlled trials are needed to evaluate whether air flow and intrapleural pressure predict clinical outcome in patients with pneumothorax and to establish guidelines for the proper management of these patients.

\section{Conclusions}

Our retrospective study proved that digital thoracic drainage devices in pneumothorax can be a valuable tool to determine the optimal timing of chest tube removal and to reduce the length of hospital stay in patients with pneumothorax. Compared to traditional analogue systems, digital drainage systems could accurately measure intrathoracic pressure, perform sufficient suction by monitoring air leaks and changes in intrathoracic pressure, and determine the optimal timing of chest tube removal without any additional procedures such as clamp tests.

\section{Acknowledgments}

Funding: This paper was supported by Konkuk University in 2020 .

\section{Footnote}

Reporting Checklist: The authors have completed the STROBE reporting checklist. Available at http://dx.doi. org/10.21037/jtd-20-2993

Peer Review File: Available at http://dx.doi.org/10.21037/jtd20-2993

Data Sharing Statement: Available at http://dx.doi. org/10.21037/jtd-20-2993

Conflicts of Interest: All authors have completed the ICMJE uniform disclosure form (available at http://dx.doi. org/10.21037/jtd-20-2993). The authors have no conflicts of interest to declare.

Ethical Statement: The authors are accountable for all aspects of the work in ensuring that questions related to the accuracy or integrity of any part of the work are appropriately investigated and resolved. The study was conducted in accordance with the Declaration of Helsinki (as revised in 2013). This study was approved by the Institutional Ethics Committee/Review Board of Konkuk University Chungju Hospital. Informed consent was waived due to its retrospective nature of this study (IRB approval No., KUCH 2019-10-031).

Open Access Statement: This is an Open Access article distributed in accordance with the Creative Commons Attribution-NonCommercial-NoDerivs 4.0 International License (CC BY-NC-ND 4.0), which permits the noncommercial replication and distribution of the article with the strict proviso that no changes or edits are made and the original work is properly cited (including links to both the formal publication through the relevant DOI and the license). See: https://creativecommons.org/licenses/by-nc-nd/4.0/.

\section{References}

1. Munnell ER. Thoracic drainage. Ann Thorac Surg 1997;63:1497-502.

2. Toth JW, Reed MF, Ventola LK. Chest Tube Drainage Devices. Semin Respir Crit Care Med 2019;40:386-93.

3. Venuta F, Diso D, Anile M, et al. Chest Tubes: Generalities. Thorac Surg Clin 2017;27:1-5.

4. Desimonas N, Tsiamis C, Sgantzos M. The Innovated "Closed Chest Drainage System" of William Smoult Playfair (1871). Surg Innov 2019;26:760-2.

5. Meyer JA. Gotthard Bülau and closed water-seal drainage for empyema, 1875-1891. Ann Thorac Surg 1989;48:597-9. 
6. Saettele TM, Jimenez CA. Digital quantification of air leak to identify the location of an alveolopleural fistula. Ann Am Thorac Soc 2014;11:1152-4.

7. Tunnicliffe G, Draper A. A pilot study of a digital drainage system in pneumothorax. BMJ Open Respir Res 2014;1:e000033.

8. Tschopp JM, Rami-Porta R, Noppen M, et al. Management of spontaneous pneumothorax: state of the art. Eur Respir J 2006;28:637-50.

9. Baumann MH, Strange C, Heffner JE, et al. Management of spontaneous pneumothorax: an American College of Chest Physicians Delphi consensus statement. Chest 2001;119:590-602.

10. Henry M, Arnold T, Harvey J, et al. BTS guidelines for the management of spontaneous pneumothorax. Thorax 2003;58:ii39-52.

11. MacDuff A, Arnold A, Harvey J, et al. Management of spontaneous pneumothorax: British Thoracic Society Pleural Disease Guideline 2010. Thorax 2010;65:ii18-31.

12. Cerfolio RJ. Advances in thoracostomy tube management. Surg Clin North Am 2002;82:833-48.

13. Anegg U, Lindenmann J, Matzi V, et al. AIRFIX: the first digital postoperative chest tube airflowmetry-a novel method to quantify air leakage after lung resection. Eur J Cardiothorac Surg 2006;29:867-72.

14. Cerfolio RJ, Bryant AS. The benefits of continuous and digital air leak assessment after elective pulmonary resection: a prospective study. Ann Thorac Surg

Cite this article as: Lee SA, Kim JS, Chee HK, Hwang JJ, Ji M, Kim YH, Moon HJ, Lee WS. Clinical application of a digital thoracic drainage system for objectifying and quantifying air leak versus the traditional vacuum system: a retrospective observational study. J Thorac Dis 2021;13(2):1020-1035. doi: 10.21037/jtd-202993
2008;86:396-401.

15. Varela G, Jiménez MF, Novoa NM, et al. Postoperative chest tube management: measuring air leak using an electronic device decreases variability in the clinical practice. Eur J Cardiothorac Surg 2009;35:28-31.

16. Brunelli A, Salati M, Refai M, et al. Evaluation of a new chest tube removal protocol using digital air leak monitoring after lobectomy: a prospective randomised trial. Eur J Cardiothorac Surg 2010;37:56-60.

17. Varela G, Brunelli A, Jiménez MF, et al. Chest drainage suction decreases differential pleural pressure after upper lobectomy and has no effect after lower lobectomy. Eur J Cardiothorac Surg 2010;37:531-4.

18. Cerfolio RJ, Varela G, Brunelli A. Digital and smart chest drainage systems to monitor air leaks: the birth of a new era? Thorac Surg Clin 2010;20:413-20.

19. Cerfolio RJ, Bryant AS. The quantification of postoperative air leaks. Multimed Man Cardiothorac Surg 2009;2009:mmcts.2007.003129.

20. McGuire AL, Petrcich W, Maziak DE, et al. Digital versus analogue pleural drainage phase 1: prospective evaluation of interobserver reliability in the assessment of pulmonary air leaks. Interact Cardiovasc Thorac Surg 2015;21:403-7.

21. Pompili C, Detterbeck F, Papagiannopoulos K, et al. Multicenter international randomized comparison of objective and subjective outcomes between electronic and traditional chest drainage systems. Ann Thorac Surg 2014;98:490-6. 

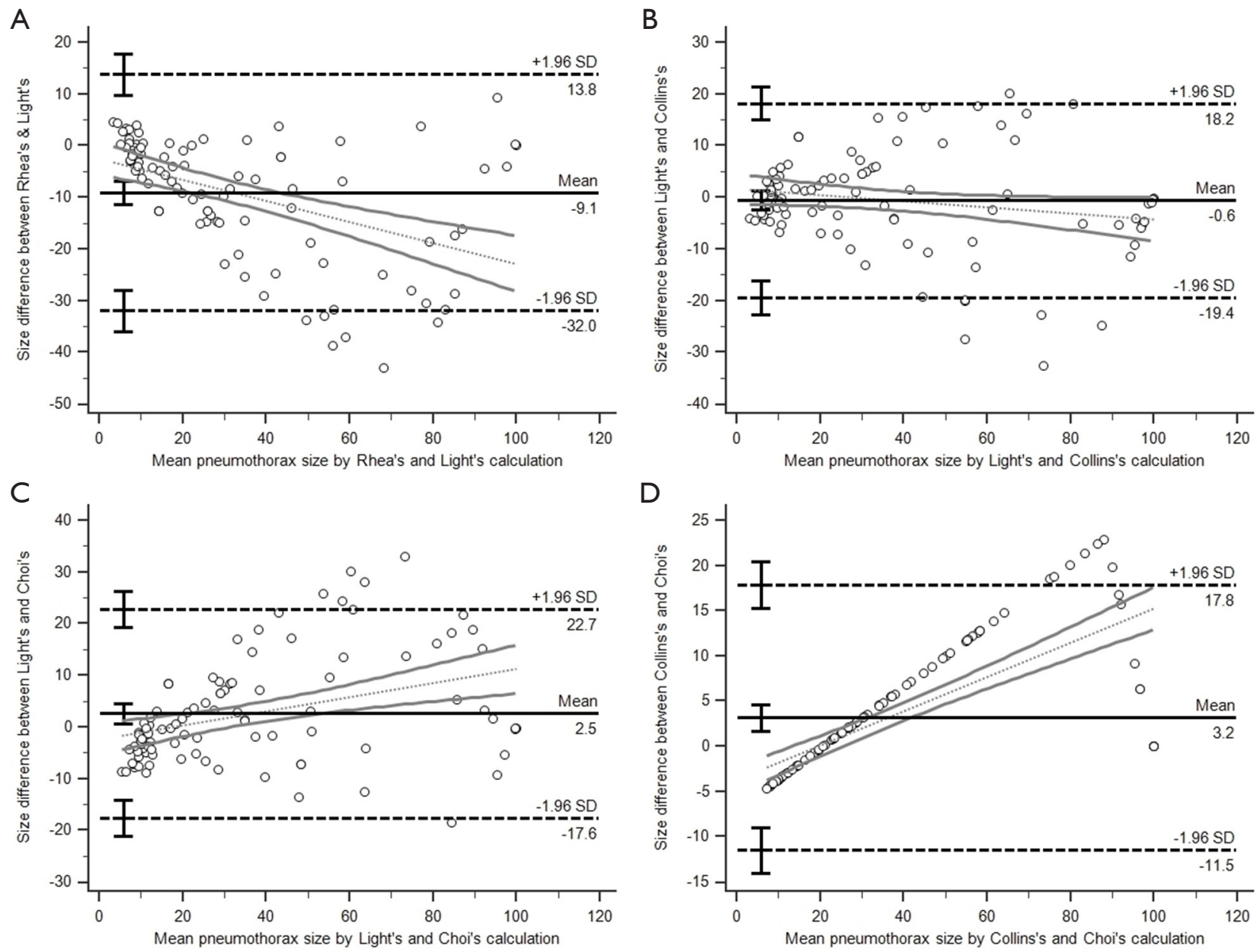

Figure S1 Bland-Altman plots show differences between the 2 methods for pneumothorax-size calculation against the averages of the 2 methods. Differences in pneumothorax size were calculated by using 4 methods of Rhea's, Light's, Collins's and Choi's calculations, based on chest PA/AP computation. In the plot, the $x$-axis means the average of the 2 evaluation methods and the $y$-axis represents the difference of the 2 evaluation methods. The thick black lines represent the mean, and the thick black dotted upper/lower lines mean the limits of agreement (LOA), defined as the mean difference \pm 1.96 standard deviation (SD) of differences. If these limits do not exceed the maximum difference between the 2 methods, the 2 methods are considered to be in good agreement and can be used interchangeably. Each standard error bar means the $95 \%$ confidence intervals (CI) of each means, upper limits of agreement and lower limits of agreement. For accurate detection of a proportional difference, the regression lines (gray dotted lines) and the $95 \%$ CI of this regression lines (double gray lines) were depicted in all figures. Only in Figure B, a scatter diagram shows a strong relationship between the data from Light's/Collins's calculations and the magnitude of measurements: differences in arithmetic mean, -0.6097 ; 95\% CI, -2.5126 to 1.2932; P=0.5264; lower limit, -19.4063 ; lower limit 95\% CI, -22.6692 to -16.1434; upper limit, 18.1869; upper limit 95\% CI, 14.9240 to 21.4498; regression equation, y=1.5948 $+(-0.05893 \mathrm{x})$. This result show that Light's method strongly depends on the magnitude of Collin's measurements. Detailed data are summarized in Tables S1. PA, posteroanterior x-ray view; AP, anteroposterior X-ray view; LOA, limits of agreement; SD, standard deviation; CI, confidence interval; $\mathrm{P}$, statistical probability. 
Table S1 Statistical analyses between the 2 methods for pneumothorax-size calculation

\begin{tabular}{|c|c|c|c|c|}
\hline Method A vs. Method B & Rhea's vs. Light's & Light's vs. Collins's & Light's vs. Choi's & Collins's vs. Choi's \\
\hline Arithmetic mean & -9.1139 & -0.6097 & 2.5449 & 3.1546 \\
\hline $95 \% \mathrm{Cl}$ & -11.4300 to -6.7978 & -2.5126 to 1.2932 & 0.5016 to 4.5882 & 1.6690 to 4.6402 \\
\hline Lower limit & -31.9924 & -19.4063 & -17.6385 & -11.5198 \\
\hline $95 \% \mathrm{Cl}$ & -35.9639 to -28.0210 & -22.6692 to -16.1434 & -21.1421 to -14.1349 & -14.0671 to -8.9725 \\
\hline Upper limit & 13.7646 & 18.1869 & 22.7283 & 17.8290 \\
\hline
\end{tabular}

$\mathrm{Cl}$, confidence interval; $\mathrm{P}$, statistical probability. 